\title{
Las simulaciones en Educación Médica
}

\author{
Jueves 25 de octubre, 12.00 horas
}

\author{
Simulation-Based Medical Education - \\ From Vision to Reality \\ Amitai Ziv, MD, MHA. \\ Director, MSR - Israel Center for Medical Simulation \\ Deputy Director, Sheba Medical Center, \\ Tel Hashomer, Israel
}

In 1999, the important report on patient safety To Err is Human was issued by the US National Institute of Medicine. It indicated that health care is far less safe than it should be, and that deaths due to medical errors in the US are over 100,000 people annually. Simulation based medical education has been recognized as a powerful tool in addressing patient safety and quality-care training as it acknowledges the adult learning concepts that experiential and immersive learning improves the absorption and retention of skills and knowledge.

Simulation in health care is used to reproduce real patient experiences/encounters with guided and controlled simulation based scenarios. It offers a safe and "mistake-forgiving" environment where trainees can learn from their errors without the risk of harming real patients. Training is learner oriented, which enables consideration of the trainees' needs, deficiencies, and their pace of learning, without the ethically disturbing use of actual patients that is associated with traditional bedside teaching. Simulation provides a hands-on empirical educational modality, enabling controlled proactive exposure of trainees to both regular and complex, uncommon clinical scenarios. This modality further supplies a unique opportunity for team training, an important contributing factor to enhance patient safety that is seldom addressed in traditional medical education. Another important benefit is the reproducible, standardized, objective setting it provides for assessment purposes.

Simulation-based medical education (SBME) is a rapidly growing field, as is illustrated by the increased development of simulation centers worldwide. The general model of most facilities focuses on a single simulation modality or a specific branch of medicine or health care, limiting their overall impact on patient safety and quality of care across the health care systems. MSR, the Israel Center for Medical Simulation, is a comprehensive, national, multimodality, multidisciplinary medical simulation center dedicated to enhancing hands-on medical education, performance assessment, patient safety, and quality of care by improving clinical and communication skills. The center uses an "error-driven" educational approach, which recognizes that errors provide an opportunity to create a unique beneficial learning experience. The 1600square-meter Center is designed as a virtual hospital and encompasses the whole spectrum of medical simulation modalities. These include simulated patients, advanced task trainers and virtual reality surgical simulators, and cutting-edge, computer-driven, full-body mannequins, that enable team training for high risk clinical conditions. MSR has trained over 30,000 trainees of various health professions in over 100 different types of courses that develop clinical and communication skills and enable team training in "mistake forgiving" environments.

The lecture will review the evolution, current status and trends in medical simulation. It will also focus on the challenges and lessons learned from the Israeli experience in operating a national simulation center since 2001. It will conclude with describing the actual and potential impact of simulation-based education on revolutionizing the educational culture and the safety culture in medicine.

\section{Modelos, estrategias y tendencias en España de la simulación en medicina Guillermo Vazquez Mata. \\ Director de I+D, Fundación IAVANTE. \\ Centro Multifuncional Avanzado para el Entrenamiento e Innovación Tecnológica. Consejería de Salud, Junta de Andalucía}

\section{La simulación como una necesidad ineludible}

La práctica clínica es la suma de un pensamiento anatómico, fisiopatológico, clínico, farmacológico y social, y que una vez integrado permite la toma de decisiones. El progreso continuo del conocimiento médico y de las tecnologías en las que se apoya, obligan a desarrollar a una estrategia de transferencia eficaz del conocimiento en tiempo, calidad y accesibilidad, junto con otra estrategia para la adquisición de las habilidades tecnológicas adecuadas al conocimiento que se quiere transferir. Esto supone para el médico y enfermero asistencial un permanente reajuste en su formación para adecuarla a los cambios de su entorno. La respuesta al panorama descrito es compleja y debe de basarse en una renovación en las metodologías de formación y entrenamiento, y la utilización de las nuevas tecnologías con un papel muy relevante para aquellas relacionadas con la Información y Comunicación (TICs).

Las metodologías sobre las que se basa esta renovación son el aprendizaje basado en problemas que promueve el pensamiento analítico y crítico, y la simulación que busca el entrenamiento de los profesionales en laboratorios de aprendizaje que reproducen las condiciones reales en las que dichos profesionales desarrollaran su actividad. La simulación tiene diversas modalidades basadas en diferentes tecnologías, la microsimulación, los maniquís humanos interactivos, la realidad virtual, y finalmente la simulación de enfermedades con actores. Las ventajas de estas metodologías radican en la mejora de las curvas de aprendizaje, tanto en su vertiente cualitativa como en el factor tiempo, junto con una mejora en las garantías de calidad para los pacientes. 


\section{La simulación en España.}

España, al igual que otros países de la UE, está incorporando la simulación al panorama educativo a través de Centros de Simulación basados en distintos modelos. Estos centros reúnen diversos requisitos; suelen ser edificios interactivos, gracias al empleo de las TICs en todos sus espacios, poseen laboratorios de entrenamiento, y salas talleres de análisis y retroalimentación; en estos espacios se encuentran los recursos de simulación que pueden dividirse en varios dos apartados: equipos para la simulación médica y equipos para la simulación en cirugía. En general los equipos médicos corresponden a maniquís que simulan alguna parte del organismo humano, y permiten el entrenamiento en habilidades manuales básicas, o bien maniquís humano completos e interactivos que permiten reproducir la semiología básica cardiorrespiratoria, así como la función cardiovascular y pulmonar completas. Estos últimos maniquís, reproducen mediante un software, cuadros clínicos diversos, que el equipo médico y enfermero deberán de identificar, y tratar. Los equipos de simulación quirúrgica suelen corresponder a equipos de realidad virtual y están dirigidos especialmente a las nuevas tecnologías quirúrgicas, soliendo combinarse zonas de entrenamiento en microcirugía, cirugía de mínima invasión y cirugía endoscópica y endovascular. Estos centros a su vez suelen apoyarse en un Campus Virtual, en los que se gestiona la parte administrativa de los cursos, a la vez que se da apoyo de eLearning, y como instrumento de comunicación y colaboración. Paralelamente se necesitan espacios para almacenes, servicios administrativos y espacios para los especialistas no médicos que diseñan las estructuras docentes de los cursos.

En España, cabe destacar cuatro experiencias que suponen distintos enfoques de la simulación, tanto en sus variantes con en su gestión. La primera es la Fundación IAVANTE, de la Consejería de Salud de Andalucía, dedicada a la transferencia del conocimiento y el entrenamiento de los profesionales de la sanidad en toda Andalucía, y que cubre tanto el entrenamiento del área médico / enfermero como la del área quirúrgica; entre su diversas sedes destaca el Centro Multifuncional Avanzado de Simulación e Innovación Tecnológica, localizado en Granada, Esta Fundación representa una apuesta de la Administración Pública Andaluza por la mejora permanente actualización de sus profesionales sanitarios. En segundo lugar el Centro de Cirugía de Mínima Invasión “Jesus Uson”, ubicado en Cáceres, y dedicado exclusivamente al entrenamiento en nuevas tecnológicas quirúrgicas, y que representa el mayor complejo de entrenamiento quirúrgico de la Unión Europa. En tercer lugar el Centro de Entrenamiento en Situaciones Críticas, localizado en Hospital Marques de Valdecilla de Cantabria, y que representa una alianza entre servicios hospitalarios e Instituciones extrahospitalarias, que ha permitido crear un servicio de simulación monotemático; es fácilmente asimilable en otros hospitales. Finalmente el Instut d'Estudis de la Salut, perteneciente a la Consejería de Salut de Cataluña, que combina una misión y objetivos sumamente diversos, entre los que se encuentran los de Evaluación y Acreditación de las competencias profesionales y en las cuales utiliza la simulación con actores para los Exámenes Clínicos Objetivos Estructurados. (ECOE).
Las estrategias de los Centros de simulación.

Los Centros dedicados exclusivamente la simulación requieren desarrollar una serie de estrategias, que pueden esquematizarse en los siguientes puntos:

Identificación de una misión, valores y unos clientes apropiados a dicha misión.

Financiación, global del centro y especifica por actividad, recurriendo a fuentes diversificadas, y asegurando su sostenibilidad Geográfica, que asegure a la accesibilidad de las personas, y minimice todos los tipos de costes.

Plantillas, buscando minimizar las plantillas estables, y buscando directores de cursos y tutores entre los profesionales clínicos en activo.

Diseño y producción de los cursos, mediante cadenas de producción diferencias

Gestión de la calidad en todas sus dimensiones, y centrada en procesos y resultados.

Innovación permanente mediante alianzas con los centros de conocimiento

\section{Barreras actuales.}

La simulación no está incorporada a los curricula de los estudiantes de medicina, ni en la formación de especialistas, por lo cual no se percibe como una necesidad

Los costes de los equipos de simulación, tanto robótica como virtual, bloquean su expansión, impidiendo alcanzar una masa crítica de cambio.

Las actividades que subyacen detrás de este tipo de acciones formativas, no pueden afrontarse individualmente, al revés de los que sucede con las clases magistrales; hospitales, y facultades de medicina requieren un rediseño de sus estructuras de apoyo a la formación.

Profesores y tutores necesitan un entrenamiento especifico en el manejo, diseño e implementación de este tipo de acciones formativas;

El tiempo que se requiere, superan con creces el de la lección magistral, siendo difícil que un médico o enfermero lo pueda dedicar.

El currículo de médicos y enfermeros recoge el esfuerzo docente como un merito secundario

No existe una infraestructura investigadora que refuerce este ámbito del conocimiento médico.

\section{Tendencias futuras:}

Las Unidades de Simulación se incorporarán a los centros hospitalarios, y centros de salud, trabajando en colaboración para evitar reduplicar esfuerzos, intercambiar experiencias, y mejorar la coordinación asistencial interniveles.

Los Centros de Simulación posiblemente se utilizarán también como Living Labs para las tecnologías sanitarias, es decir los mismos espacios y diseños de entrenamiento, se dedicarán a mejorar el empleo de todo tipo de equipo tecnológico antes de aplicarse en la realidad.

Se formaran Clusters del Conocimientos, es decir, se trabajará en proyectos innovadores en alianza con instituciones tipo escuelas de ingenieros e industrias, para generar patentes, prototipos industriales, o compañías tipo spinoff.

La seguridad del paciente, y sus posibles repercusiones legales, potenciará el empleo de la simulación. 